\title{
Digitale Gestaltung innovativer \\ Gesundheitsnetzwerke - Erfolgreiches \\ Netzwerkmanagement im Gesundheits- \\ und Dienstleistungssektor
}

\author{
Christoph Buck, Simone Burster, Serkan Sarikaya, Julia Thimmel \\ und Torsten Eymann
}

\section{Inhaltsverzeichnis}

23.1 Netzwerke als Möglichkeit zur verbesserten Versorgung im Gesundheitswesen . . . . . 448

23.2 Netzwerkmanagement im Gesundheitswesen als Erfolgsfaktor . . . . . . . . . . . . 450

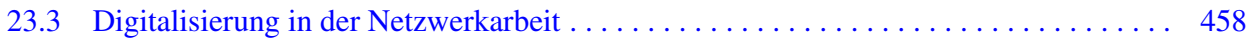

23.3.1 Netzwerkinfrastruktur - Möglichkeiten zur digitalen Unterstützung der

Basis für das Netzwerkmanagement . . . . . . . . . . . . . . . . 460

23.3.2 Netzwerkgovernance - Vereinfachte Steuerung des Netzwerks durch technologische Unterstützung $\ldots \ldots \ldots \ldots \ldots \ldots \ldots \ldots \ldots \ldots \ldots \ldots \ldots \ldots$

C. Buck $(\triangle) \cdot$ S. Burster $\cdot$ T. Eymann

Rechts- und Wirtschaftswissenschaftliche Fakultät, Lehrstuhl Wirtschaftsinformatik,

Universität Bayreuth, Bayreuth, Deutschland

E-Mail: christoph.buck@uni-bayreuth.de

S. Burster

E-Mail: simone.burster@uni-bayreuth.de

T. Eymann

E-Mail: torsten.eymann@uni-bayreuth.de

S. Sarikaya

Kernkompetenzzentrum Finanz- \& Informationsmanagement, Kernkompetenzzentrum,

Augsburg, Deutschland

E-Mail: serkan.sarikaya@fim-rc.de

J. Thimmel

, Universität Bayreuth, Bayreuth, Deutschland

E-Mail: julia.thimmel@gmx.de 
23.3.3 Netzwerkmoderation - Koordinierung und operative Organisation vereinfachen............................... 463

23.3.4 Netzwerkpromotion - Außendarstellung durch digitale Medien verbessern. . . 467

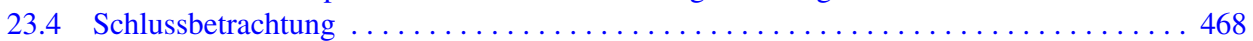

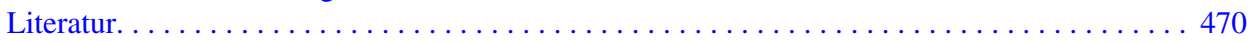

\section{Zusammenfassung}

Die Gesundheitsversorgung in Deutschland steht durch die alternde Bevölkerung und sektorale Trennung der Leistungserbringer vor großen Herausforderungen. Um auch zukünftig den Bedarf an gesundheitlichen, medizinischen und pflegerischen Leistungen decken zu können, benötigt es neue Versorgungskonzepte. Ein Lösungsansatz dafür können Netzwerke als Organisationsform sein, um die verschiedenen Leitungserbringer zusammenzubringen und bessere gesundheitliche Versorgung leisten $\mathrm{zu}$ können. Dabei kann auch die Digitalisierung wertvollen Zusatznutzen bringen. Durch digitale Vernetzung können im Netzwerkmanagement noch mehr Potenziale genutzt und die Netzwerkarbeit unterstützt und optimiert werden. Der Beitrag zeigt auf, wie Netzwerkmanagement sinnvoll anhand der vier Kernfunktionen Netzwerkinfrastruktur, Netzwerkgovernance, Netzwerkmoderation und Netzwerkpromotion nachhaltig etabliert und effizient gestaltet werden kann.

\subsection{Netzwerke als Möglichkeit zur verbesserten Versorgung im Gesundheitswesen}

Das deutsche Gesundheitswesen ist mit einer Bruttowertschöpfung von 369,8 Mrd. € einer der größten Wirtschaftssektoren in Deutschland, welcher stetig weiterwächst. Dabei ist die Gesundheitswirtschaft seit 2007 nominal angestiegen. Der Anteil der Gesundheitswirtschaft an der Gesamtwirtschaft ist im Zeitverlauf von 10,5 \% im Jahr 2007 auf 12,1 $\%$ im Jahr 2018 gewachsen. (BMWi 2019).

Allerdings steht das Gesundheitswesen vor erheblichen strukturellen Herausforderungen. Aufgrund des demografischen Wandels in Deutschland erfolgt eine Entwicklung hin zu einer immer älter werdenden Gesellschaft (Robert Koch-Institut 2018). Durch den medizinischen Fortschritt und innovative Entwicklungen in der Krankenbehandlung kann die Alterung der Gesellschaft besser behandelt werden (Braeuninger und Rakau 2010). Dadurch steigt die Lebenserwartung in der Bevölkerung. Andererseits steigt auch die Wahrscheinlichkeit der Multimorbidität. Durch das gleichzeitige Bestehen mehrerer Krankheiten im Alter wachsen der Versorgungsaufwand im Gesundheitswesen und die Nachfrage nach Ärzten und weiterem Fachpersonal in der Branche (Braeuninger und Rakau 2010). Circa ab dem 35. Lebensjahr steigen die Gesundheitsausgaben mit dem Alter exponentiell an und flachen erst im hohen Alter wieder etwas ab. Aufgrund der Alterung der Gesellschaft befinden sich mehr Personen in der Altersgruppe, in welcher höhere Ausgaben, z. B. aufgrund der Multimorbidität, verursacht werden. Somit werden die Gesundheitsausgaben immer mehr in die Höhe getrieben. Zusätzlich werden die 
Kostenentwicklungen durch die steigende Lebenserwartung der Bevölkerung verschärft. Statistiken zeigen auf, dass die Gesamtbevölkerung in Deutschland ein immer höheres Alter erreicht (Statista 2018). Zudem besteht in einzelnen Bereichen der medizinischen Versorgung eine Überversorgung (Aumiller 2014; Stiefelhagen 2018), beispielsweise in ambulanten Behandlungen bei der Diabetesbehandlung von älteren Patienten (Stiefelhagen 2018). Durch überflüssige diagnostische und therapeutische Maßnahmen werden hohe Kosten als unnötige Belastung für den Patienten und das Solidarsystem generiert (Fiedler 2018). Dabei besteht ein enger Zusammenhang bezüglich der fehlenden Vernetzung zwischen den Sektorengrenzen. Diese Entwicklungen zeigen die finanzielle Belastung des Gesundheitssystems auf sowie einen zunehmenden Effizienzdruck.

Ein weiteres strukturelles Problem, das im Gesundheitswesen vorherrscht, ist die bestehende sektorale Trennung zwischen der ambulanten und der stationären Leistungserbringung (Gerlinger und Burkhardt 2018). Durch fehlende Absprachen zwischen den Leistungserbringern aus den unterschiedlichen Sektoren bestehen Schnittstellenproblematiken, wie Koordinations-, Kommunikations- oder Kooperationsprobleme. Aufgrund von Informationsdefiziten oder Unwissenheit von Verantwortlichkeiten können schlechtere Behandlungsergebnisse die Folge sein. Dies kann bei sektorenübergreifenden Behandlungen aufgrund des fehlenden Austauschs zu Unwirtschaftlichkeit, Ineffizienzen und unnötigen Aufwänden in der Behandlung führen. Oftmals wirkt sich dies, gerade in komplexen Fällen, auch negativ auf die Behandlungsqualität aus (Bönisch 2017).

Erschwerend hinzu kommt, dass im deutschen Gesundheitswesen eine sehr heterogene Systemlandschaft besteht (Aschhoff 2017). Beispielsweise werden in Arztpraxen andere Patientenverwaltungssysteme verwendet als in physiotherapeutischen Praxen. Selbst zwischen einzelnen Arztpraxen werden unterschiedliche Systeme angewendet, wodurch der Informationsaustausch zwischen den Systemen und weiteren Teilnehmern, wie beispielsweise Krankenhäusern, Krankenkassen oder anderen Dienstleistern im Gesundheitswesen, deutlich erschwert wird.

Zusätzlich beeinträchtigt wird die Inkompatibilität der Informationssysteme durch die Anforderungen an die Informationsübermittlung. Um Informationen von Diagnosen lesbar zu machen, muss eine Codierung der Diagnosen nach dem Fallpauschalensystem erfolgen. Diese Codes wurden hauptsächlich für Abrechnungszwecke entwickelt und enthalten folglich nicht die kompletten Informationen über die Diagnose, den Behandlungsvorgang und die weiteren Maßnahmen (Aschhoff 2017).

In Deutschland stellen gerade in den ländlichen Regionen diese strukturellen Probleme im Gesundheitssektor noch größere Herausforderungen dar. In ländlichen Gebieten besteht oftmals ein Versorgungsmangel, weshalb Fachkliniken und Arztpraxen häufig geschlossen werden müssen. Diese können selbst durch Intensivierungen, wie Bezuschussungen für Arztpraxen in ländlichen Regionen oder Stipendien für zukünftige Ärzte in gering besiedelten Gebieten, nicht verhindert werden (Beermann 2017). Durch den Ärztemangel und die weit zurückzulegenden Strecken in ländlichen Gebieten ist eine angemessene medizinische Versorgung noch schwieriger und es bedarf noch besserer Strukturen und digitaler Unterstützung, um dem entgegenzuwirken (Beermann 2017). 
Die aufgezeigten Problematiken im Gesundheitswesen haben alle Implikationen auf die Kostenstruktur und -höhe. So stehen die Gesamtausgaben des Gesundheitswesens immer wieder in der Debatte (Simon 2011). Der vorhandene Ausgabenanstieg wird dabei als überproportional angesehen und damit ein zu schnelles Wachstum verzeichnet (Wasmuth 2013). Durch die steigenden Kosten und den zunehmenden Fachkräftemangel entsteht immer größerer Effizienzdruck in der Branche.

Aufgrund der dargelegten Herausforderungen sind neue Ansätze für die Versorgungsstrukturen gefragt. Um sich an die verändernden gesellschaftlichen und bestehenden regional unterschiedlichen Strukturen anzupassen, können Netzwerke als Organisationsform besonders vielversprechend sein. Ziel sollte sein, Netzwerkstrukturen zwischen den unterschiedlichen Leistungserbringern zu bilden, um dadurch Synergien zu schaffen und die Versorgung zu verbessern (Becke et al. 2016). Hierbei gilt es, gezielt vorzugehen, um die Potenziale der Netzwerkarbeit optimal nutzen zu können und eine erfolgreiche Umsetzung zu gewährleisten. Dafür gilt es, ein strukturiertes und effizientes Netzwerkmanagement zu etablieren (Weatherly 2013). Durch vernetzte Strukturen können unterschiedliche Akteure, wie beispielsweise Patienten, Ärzte oder Angehörige, profitieren. Mit dem Zusammenschluss der Akteure und der damit verbundenen Einführung eines standardisierten Systems werden die Datenübermittlung und der Datenaustausch vereinfacht. Es werden Schnittstellenproblematiken verringert und die Kommunikation der unterschiedlichen Leistungserbringer verbessert. Zudem erfolgt eine verbesserte Prozessqualität und durch eine verkürzte Behandlungsdauer eine Zeitersparnis für alle Beteiligten. Insgesamt lässt sich durch die Vernetzung von Akteuren im Gesundheitswesen eine Optimierung in der Struktur, den Prozessen und dem Ergebnis erwarten (Ernstberger et al. 2017).

Neben der Vernetzung der unterschiedlichen Akteure im Gesundheitswesen stellt die Digitalisierung große Potenziale für eine verbesserte Versorgung im Gesundheitswesen dar (Bouée und Schaible 2015). Durch digitale Vernetzung kann der Austausch in Netzwerken zusätzlich optimiert werden und die Netzwerkstrukturen- und -prozesse können optimal unterstützt werden.

Um ein stärkeres gemeinsames Handeln zu ermöglichen, sind die Entwicklung und die Etablierung erfolgreicher Netzwerkstrukturen und -prozesse essenziell. Dabei kann das Netzwerkmanagement anhand von vier Kernfunktionen Netzwerkinfrastruktur, Netzwerkgovernance, Netzwerkmoderation und Netzwerkpromotion gesteuert werden (Stadtelmann et al. 2015; Stabell und Fjeldstad 1998).

\subsection{Netzwerkmanagement im Gesundheitswesen als Erfolgsfaktor}

Im deutschen Gesundheitssystem gibt es zahlreiche, unterschiedliche Akteure, die es gilt, intelligent miteinander zu vernetzen, um die Herausforderungen und Hürden in der Versorgung sinnvoll zu adressieren. Dafür stellen Netzwerke, im Sinne sogenannter Wertnetze, eine geeignete Organisationsform dar (Stabell und Fjeldstad 1998). Durch 
Netzwerkstrukturen wird ermöglicht, verschiedene Akteure zusammenzubringen, um dadurch beispielsweise Kosten einzusparen, Kompetenzen zu bündeln und Synergien zu erzeugen. Dabei entsteht der Wert bei der Erbringung von Dienstleistungen nicht durch die Verkettung von Arbeitsschritten, sondern durch die Vernetzung und Koordination der Netzwerkmitglieder. Mit einer steigenden Anzahl an Netzwerkmitgliedern und somit einer größeren Nachfrage nach den Leistungen des Netzwerkmanagements werden positive Netzwerkeffekte generiert und es wird Mehrwert geschaffen (Stabell und Fjeldstad 1998; Katz und Shapiro 1985). Die Wertschöpfungsaktivitäten innerhalb des Netzwerkes liegen dabei auf der Organisation des Netzwerkes und dem Austausch im Kreise der Netzwerkmitglieder. Das Netzwerkmanagement übernimmt hierbei die Wertschöpfungsaktivitäten und somit die Vermittlungssfunktion im Netzwerk (Stadtelmann et al. 2015; Stabell und Fjeldstad 1998).

Somit wird beispielsweise in einem Tumornetzwerk, welches die sektorenübergreifende Therapie von Tumorerkrankungen behandelt, der Behandlungsprozess gezielt über die unterschiedlichen Leistungserbringer hinweg gesteuert. Dies kann zu einer Verkürzung der Behandlungsdauer führen und somit dem Patienten eine bessere Versorgung ermöglichen. Aber auch im Bereich der Gesundheitsprävention können Netzwerkstrukturen sinnvoll eingesetzt werden. Beispielsweise können in Kindergärten und Schulen Gesundheits- Jugend- und Bildungsakteure vernetzt werden, um nachhaltig die körperliche und seelische Gesundheit sowie die soziale Teilhabe bei Kindern zu verbessern (Präventionsnetzwerk Ortenaukreis 2018).

Ziel des Zusammenschlusses unterschiedlicher Akteure ist es, gemeinsam zu handeln und Kompetenzen zu bündeln. Jedoch stellt dies eine komplexe Aufgabe dar, die gewisse Herausforderungen mit sich bringt, da die deutsche Gesundheitsbranche durch eine wechselseitige, historisch gewachsene Abschottung der Versorgungsbereiche geprägt ist. Dadurch ist die Vernetzung unterschiedlicher Akteure besonders herausfordernd und komplex. Deshalb ist es grundlegend für das Netzwerkmanagement und für die erfolgreiche und nachhaltige Entwicklung und Etablierung von Netzwerkstrukturen und -prozessen, die richtigen Ressourcen als auch Kompetenzen, die für die Netzwerkarbeit benötigt werden, einzusetzen. Im Sinne einer reflexiven Netzwerkentwicklung entfalten sich Netzwerke durch ein kontinuierliches Wechselspiel aus Veränderungen und Anpassungen innerhalb der Netzwerke sowie im Austausch mit ihren Umfeldern (Sydow 2010). Um daher das Netzwerkmanagement erfolgreich gestalten zu können, werden Konzepte, Maßnahmen sowie Ressourcen entlang von vier zentralen Funktionen benötigt: der Netzwerkinfrastruktur, der Netzwerkgovernance, der Netzwerkmoderation und der Netzwerkpromotion (Stadtelmann et al. 2015; Stabell und Fjeldstad 1998). Für den nachhaltigen Erfolg des Netzwerks ist ein harmonisches Zusammenspiel dieser vier Funktionsbereiche unabdingbar.

\section{Die vier Kernfunktionen des Netzwerkmanagements}

Die Kernfunktionen Netzwerkinfrastruktur, Netzwerkgovernance, Netzwerkmoderation und Netzwerkpromotion repräsentieren jeweils einen übergeordneten Funktionsbereich 
des Netzwerkmanagements, der die relevanten operativen Aufgaben der Kernfunktion umfasst. Zur Umsetzung dieser vier Funktionsbereiche ist der Einsatz eines Netzwerkmanagements mit den notwendigen Ressourcen und Kompetenzen erforderlich. In Abb. 23.1 werden die vier zentralen Funktionen mit ihren Kernaufgaben dargestellt.

Die Basis für das Netzwerkmanagement bildet die gemeinsame Netzwerkinfrastruktur. Diese umfasst die Verwaltung der finanziellen, personellen und physischen Ressourcen sowie die gemeinsame IT-Strategie inklusive einer Auswahl geeigneter Informationssysteme und Kommunikationskanäle (Fitterer und Rohner 2010; Boughzala und Bououd 2013; Tapia 2009; Blondiau et al. 2013). Sie ist somit die Grundlage für die Netzwerkarbeit und Voraussetzung für den Aufbau und den Betrieb des Netzwerkes (Stadtelmann et al. 2015). Ohne grundlegende finanzielle Ressourcen ist ein nachhaltiger Aufbau eines Netzwerks nur schwer möglich. Nur durch ausreichend zur Verfügung stehende finanzielle Mittel kann bspw. Personal eingestellt werden, das wiederum Projekte und Veranstaltungen durchführt sowie den Bekanntheitsgrad des Netzwerks durch Öffentlichkeitsarbeit steigert. Durch Interviews mit unterschiedlichen Netzwerken aus der Praxis hat sich gezeigt, dass Netzwerkmanagement nur mit hauptamtlich beschäftigtem Personal in professioneller und damit effektiver Weise leistbar ist. Daher ist es wichtig, entsprechendes Personal für die Bewältigung der vielfältigen Aufgaben des Netzwerkmanagements zu beschäftigen. Die Mitarbeiter des Netzwerkmanagements sollten dabei gute koordinative und kommunikative Fähigkeiten aufweisen. Je nach inhaltlicher Ausrichtung und Strategie des Netzwerks kann die Beschäftigung weiterer Mitarbeiter (z. B. medizinische Fachangestellte) im Netzwerk sinnvoll sein.

\begin{tabular}{|l|}
\hline \multicolumn{1}{|c|}{ Moderation } \\
\hline - Rollenverständnis \& \\
Verantwortung \\
- Intensität der \\
Zusammenarbeit \\
- Kommunikation \\
\& Zusammenarbeit \\
- Serviceangebot für \\
Netzwerkmitglieder \\
- Netzwerkkultur \\
\hline
\end{tabular}

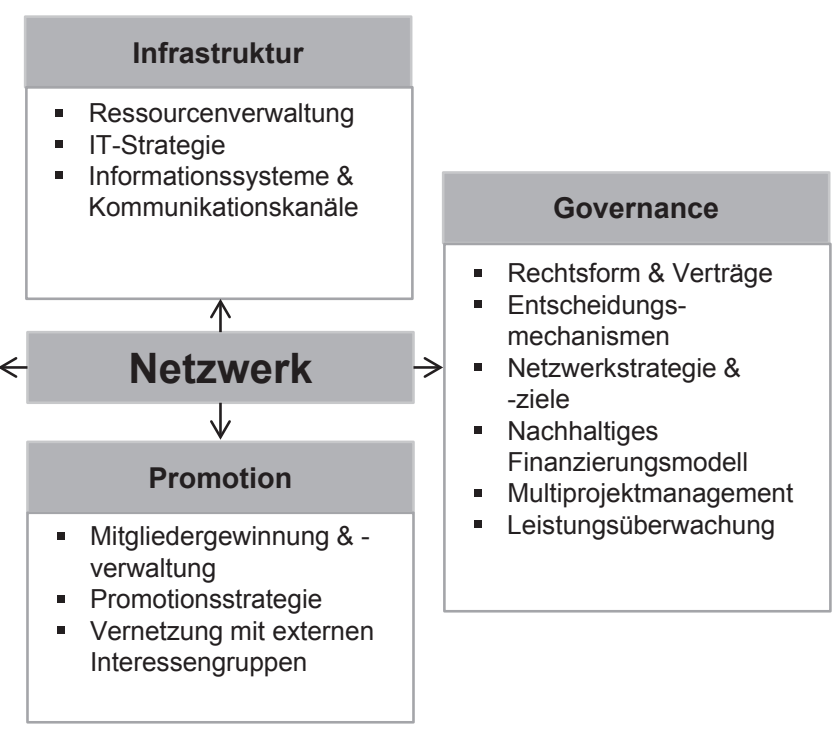

Abb. 23.1 Kernfunktionen des Netzwerkmanagments. (Quelle: eigene Darstellung 2018) 
Neben der Bereitstellung von Ressourcen zählt auch eine sinnvolle Ressourcenallokation innerhalb des Netzwerks zur Netzwerkinfrastruktur. Operativ wird diese Rolle meist durch den Geschäftsführer des Netzwerks (Netzwerkmoderation) durchgeführt. Es wird darauf geachtet, welche Mitglieder welche Zuständigkeiten und Verantwortlichkeiten haben und wie die Aufgaben entsprechend der Fähigkeiten und Kapazitäten der Netzwerkmitglieder zugeteilt werden (Sydow und Duschek 2013).

Als physische Ressourcen sind beispielsweise Büro- und Besprechungsräume für die Netzwerkarbeit erforderlich. Hierfür sind adäquate Räumlichkeiten anzumieten. Teilweise können Netzwerke auch Räume öffentlicher Einrichtungen (z. B. der Kommune, der Stadt oder der Universitätsklinik) oder anderer Kooperationspartner kostenfrei mitnutzen. In seltenen Fällen ist der Erwerb eigener Immobilien sinnvoll. Nur bei sehr etablierten und langfristig ausgelegten Netzwerken (z. B. in Ärztenetzen für die regionale Gesundheitsversorgung) ist dies eine denkbare Alternative.

Hinsichtlich der IT-Unterstützung der Netzwerkarbeit gilt es, eine gemeinsame Strategie festzulegen (Fitterer und Rohner 2010; Blondiau et al. 2013). Bei Gesundheitsnetzwerken geht es primär um die Abwägung zwischen einer einheitlichen IT-Lösung zur Ermöglichung einer elektronischen Patientenakte auf der einen Seite und dem Einsatz einer Vernetzungssoftware für heterogene IT-Systeme der einzelnen Mitglieder auf der anderen Seite. In der Praxis zeigt sich, dass die IT-Ausstattung (z. B. KV-SafeNet-Anschluss bei Ärztenetzen) Voraussetzung zur Aufnahme in ein Netzwerk ist. Während die einheitliche IT-Lösung mit hohen Investitionen für die einzelnen Mitglieder einhergeht, stärkt sie den Zusammenhalt im Netzwerkverbund, steigert die Effizienz der Netzwerkarbeit und trägt darüber hinaus zur besseren Versorgung von Patienten bei. Abgesehen von Ärztenetzwerken wird jedoch oftmals an heterogenen IT-Systemen festgehalten. Teilweise kommt eine Vernetzungssoftware für den Datenaustausch zum Einsatz, welche die kostengünstigere Variante ist und den Mitgliedern mehr Flexibilität und Freiräume bietet. Die Entscheidung für ein IT-System für die Vernetzung der Mitglieder sollte an der übergreifenden Netzwerkstrategie ausgerichtet sein. Grundsätzlich sollten IT-Systeme immer dort eingesetzt werden, wo sie die Arbeit entlasten, Prozesse beschleunigen, Fehler reduzieren, Kommunikation erleichtern und generell die Effizienz steigern (z. B. Software für die vereinfachte Dokumentation und Berichterstellung). Die Netzwerkinfrastruktur umfasst darüber hinaus die Bereitstellung der erforderlichen und nützlichen Kommunikationskanäle, um die Mitglieder zu vernetzen. Damit die Kommunikationskanäle (z. B. Videokonferenzsystem, Intranet, E-Mail) von den Mitgliedern und dem Management angenommen werden, ist es wichtig, diese an den Bedürfnissen und Präferenzen der Beteiligten auszurichten, danach auszuwählen und klare Vorgaben für die Nutzung festzulegen (Fitterer und Rohner 2010; Boughzala und Bououd 2013; Tapia 2009; Blondiau et al. 2013).

Aufbauend auf der Netzwerkinfrastruktur bildet die Netzwerkgovernance die strategische Ebene des Netzwerkmanagements ab. Die Kernaufgaben der Netzwerkgovernance umfassen die Institutionalisierung von Steuerungsformen und -mechanismen. Diese orientieren sich an der Organisationsstruktur des Netzwerks und sind daher nicht 
streng hierarchisch, sondern weisen eine dezentrale Verantwortungsstruktur auf (Schubert 2008). Im Allgemeinen beschreiben Governance-Strukturen das institutionelle Zusammenwirken verschiedener Akteure und umfassen institutionelle Vereinbarungen, die die Beziehungen zwischen den einzelnen Stakeholdern kontrollieren und diese steuern (Gelauff und Broeder 1997). Zentrale Einflussfaktoren auf die Netzwerkgovernance sind beispielsweise Gesetze, Satzungen und Regulierungsvorschriften. Diese formellen Aspekte prägen die Zusammenarbeit im Netzwerk ebenso wie informelle Elemente (z. B. Mission Statement, Vision des Netzwerkes). Darüber hinaus hat die Netzwerkgovernance die Regulationsfunktion im Netzwerk und umfasst deshalb die Festlegung und Implementierung der formellen und informellen Regeln der Netzwerkzusammenarbeit (Sydow und Duschek 2013). Daher stellen die Auswahl einer geeigneten Rechtsform für das Netzwerk und die vertraglichen Regelungen der Zusammenarbeit wichtige Aufgaben dar, die insbesondere zu Beginn der gemeinsamen Netzwerkarbeit festgelegt werden müssen (Blondiau et al. 2013; Boughzala und Bououd 2013). Wichtig ist hierbei, dass die Rechtsform zu den Netzwerkzielen und den Netzwerkaktivitäten passt und gegebenenfalls die Abwicklung wirtschaftlicher gewinnbringender Vorgänge ermöglicht. Weiterhin müssen die Rechte und Pflichten von Mitgliedern, Kooperationspartnern und dem Management vertraglich geregelt werden, damit Verantwortlichkeiten klar zugewiesen werden können und die Netzwerkarbeit in verlässlichen Strukturen ablaufen kann (Boughzala und Bououd 2013). Obwohl Netzwerke aufgrund ihrer Organisationsstruktur nicht streng hierarchisch aufgebaut sind, gilt es im Rahmen der Netzwerkgovernance, Entscheidungsmechanismen und Führungsgremien, wie beispielsweise einen Beirat oder Aufsichtsrat, zu etablieren (Srai et al. 2013; Cuenca et al. 2013; Fitterer und Rohner 2010). Zur Sicherstellung richtiger Entscheidungen ist es wichtig, dass es klar definierte Prozesse und Entscheidungsbefugnisse im Netzwerk gibt (Blondiau et al. 2013). Beispielsweise kann der Prozess zur Aufnahme neuer Mitglieder dadurch geregelt sein, dass ein formaler Mitgliedsantrag gestellt wird, welcher dann durch den Vorstand des Netzwerks genehmigt werden muss. Die strategische Auswahl der potenziellen Netzwerkmitglieder sollte immer nach Kompetenzen und der fachlichen Übereinstimmung erfolgen.

Ein zentraler Aspekt der Netzwerkgovernance ist die Entwicklung einer übergreifenden Netzwerkstrategie mit Einbeziehung aller relevanten Rahmenbedingungen und Interessengruppen, die die Netzwerkarbeit beeinflussen (Boughzala und Bououd 2013; Fitterer und Rohner 2010; Srai et al. 2013). Ein erfolgreiches Netzwerk braucht Strategien und Ziele, die von allen Mitgliedern getragen werden und auf die gemeinschaftlich hingearbeitet wird.

Zur Strategieentwicklung zählt auch ein nachhaltiges Finanzierungsmodell für die Netzwerkarbeit (Fitterer und Rohner 2010; Srai et al. 2013). Das Netzwerk kann sich mittel- und langfristig nur entwickeln und einen positiven Beitrag leisten, wenn sein Fortbestehen durch geeignete Einnahmequellen gesichert ist. Dabei gibt es verschiedene Möglichkeiten zur Finanzierung der Netzwerkarbeit, angefangen von öffentlichen Fördermitteln über Drittmittelprojekte, Kapitaleinlagen der Mitglieder und Mitgliedsbeiträge bis hin zu Einnahmen aus eigenen wirtschaftlichen Aktivitäten (z. B. kostenpflichtige 
Dienstleistungen). Dabei ist zu berücksichtigen, dass die Verwendung der vorhandenen Ressourcen immer an der Netzwerkstrategie ausgerichtet werden sollte, genauso wie die Auswahl der relevanten Themen für die Netzwerkarbeit und die Auswahl der durchzuführenden Projekte (Fitterer und Rohner 2010; Srai et al. 2013). Neben der Auswahl geeigneter Projekte für das Netzwerk gehört auch die Steuerung dieser zum Aufgabenbereich der Netzwerkgovernance. Durch geeignete Projektmanagementmaßnahmen und die Durchführung einer Leistungsüberwachung von Mitgliedern und Management können Projekte im Netzwerk besser gelenkt werden (Fitterer und Rohner 2010; Srai et al. 2013; Tiemeyer 2017; Schwarze 2014). Dabei gilt es für das Netzwerkmanagement in seiner Governance-Funktion, den Überblick über alle laufenden Projekte zu behalten.

Neben der Steuerungsfunktion hat die Netzwerkgovernance auch eine Evaluationsfunktion und adressiert die Fragen der Kosten und des Nutzens der Netzwerkarbeit im Allgemeinen und der Netzwerkbeziehungen im Speziellen (Sydow und Duschek 2013). Daher ist es zur besseren Leistungsüberwachung hilfreich, die Projektfortschritte mit geeigneten Kennzahlen zu verfolgen und Wechselwirkungen zwischen einzelnen Projekten (z. B. personelle Restriktionen, notwendige Ergebnisse für nachgelagerte Projekte) zu berücksichtigen (Beer et al. 2015; Tiemeyer 2017). Neben der Überwachung der Projektfortschritte müssen auf einer übergeordneten Ebene auch die Erreichung der Netzwerkziele und die Leistungserbringung von Mitgliedern und Management kontrolliert und dokumentiert werden. Die Erkenntnisse aus der Leistungsüberwachung, die sich auf das gesamte Netzwerk beziehen können oder auch auf einzelne Mitglieder und deren Leistungsbeiträge, fließen idealerweise wieder in strategische Überlegungen ein (Schwarze 2014).

Eine weitere zentrale Funktion des Netzwerkmanagements stellt die Netzwerkmoderation dar. Die Netzwerkmoderation repräsentiert das operative Management und ist zuständig für alle internen und externen Anfragen. Die Aufgaben der Netzwerkmoderation werden durch die Rolle des Netzwerkmoderators (dieser kann durch eine oder mehrere Personen repräsentiert sein) übernommen. Dieser ist Anlaufstelle für alle Netzwerkmitglieder als auch Kooperationspartner und koordiniert die Kommunikation und die Zusammenarbeit im Netzwerk (Cuenca et al. 2013; Boughzala und Bououd 2013; Tapia 2009). Somit repräsentiert der Netzwerkmoderator eine neutrale Unterstützung der Kommunikationsprozesse und durch den Moderator werden alle Aufgaben des Netzwerks organsiert.

Als zentrale Rolle in der Netzwerkarbeit hat die Netzwerkmoderation die gemeinsam erarbeiteten Zielvereinbarungen im Blick und forciert die Netzwerkarbeit entlang der strategischen Ausrichtung des Netzwerks, die durch die Netzwerkgovernance festgelegt wird. Der Netzwerkmoderator achtet darauf, die Aufgaben und Prozesse immer wieder auf diese zu sensibilisieren und darauf auszurichten. Damit verbunden sind das Unterbreiten von Vorschlägen zur Strukturierung von Arbeitsprozessen, die Integration von unterschiedlichen Interessen der Netzwerkmitglieder sowie die Klärung und im besten Fall die Unterbindung von Konflikten. Daher umfasst die Netzwerkmoderation auch die Rolle eines Mediators im Netzwerk. Um diese Aufgabe optimal umzusetzen, ist es 
wichtig, dass die Rolle der Netzwerkmoderation durch eine Person mit hohen sozialen Fähigkeiten besetzt ist, die zugleich empathisch ist. Sie muss ein Gespür für die einzelnen Interessen der Mitglieder haben als auch für die Stimmung im Netzwerk (Teller und Longmuss 2007). Regelmäßige Mitgliederbefragungen helfen die Stimmungslage zu erfassen und darüber hinaus zu kontrollieren.

Um Synergien für alle Mitglieder zu schaffen, werden im Rahmen der Netzwerkmoderation Shared Services, also Dienstleistungen für die Netzwerkmitglieder, angeboten, um diese in ihrer Arbeit zu entlasten (Blondiau et al. 2013). Daher umfasst die Netzwerkmoderation in erster Linie organisatorische Aufgaben und Verwaltungsaufgaben, um die Mitglieder zu entlasten, damit diese sich mehr auf ihre Kernaufgaben konzentrieren können. Auf Basis von Bedarfsanalysen im Mitgliederkreis gilt es, Dienstleistungen für die Mitglieder anzubieten (Blondiau et al. 2013). Beispiele hierfür können sein die Organisation von Fort- und Weiterbildungen für die Mitarbeiter des Netzwerks oder die zentralisierte und systematische Verfolgung von Projektausschreibungen. Darüber hinaus umfasst die Netzwerkmoderation auch Maßnahmen zur Motivation der Mitglieder zur aktiven Mitarbeit im Netzwerk und zur Entwicklung einer wertstiftenden Netzwerkkultur (Cuenca et al. 2013; Boughzala und Bououd 2013; Blondiau et al. 2013; Srai et al. 2013).

Damit die Netzwerkarbeit strukturiert, effizient und effektiv ablaufen kann, werden Prozesse und klare Kommunikationsregeln benötigt. Im Rahmen der Netzwerkmoderation werden diese Regeln für den Ablauf festgelegt. Durch regelmäßige Treffen und Abstimmungen sowie unterschiedliche Maßnahmen, wie zum Beispiel das Bilden von Arbeitsgruppen oder die Organisation von Veranstaltungen, soll auf die Erreichung der Netzwerkziele hingearbeitet werden. Die Definition von Kommunikationskanälen für bestimmte Zwecke (z. B. E-Mails für Erfahrungsberichte oder Mitgliederversammlungen für Abstimmungen) soll die Kommunikation im Netzwerk standardisieren und einen zielgerichteten Informationsfluss ermöglichen (Cuenca et al. 2013; Fitterer und Rohner 2010; Boughzala und Bououd 2013; Tapia 2009; Srai et al. 2013; Blondiau et al. 2013). Die im Rahmen der Netzwerkmoderation festgelegten Aufgabenbereiche können nur sinnvoll umgesetzt werden, wenn Rahmenbedingungen des Netzwerks so ausgestaltet sind, dass das operative Management genügend Durchsetzungsvermögen, Akzeptanz und Autorität bei den Mitgliedern hat.

Die Kernfunktion mit der höchsten Wirkung nach außen, die somit mit der Öffentlichkeitsarbeit und der Wahrnehmung des Netzwerks durch Dritte assoziiert wird, wird durch die Netzwerkpromotion verkörpert. Diese enthält alle Aufgaben mit Außenwirkung (z. B. einheitliches Corporate Design, Messeaufritte des Netzwerks, Internetaufritt), aber auch die Verwaltung und Gewinnung der Netzwerkmitglieder (Fitterer und Rohner 2010; Boughzala und Bououd 2013; Tapia 2009). Um das Netzwerk bestmöglich nach außen zu repräsentieren, gilt es, unter Berücksichtigung der übergreifenden Netzwerkstrategie, eine Promotionsstrategie für das Netzwerk zu entwickeln (Sydow und Duschek 2013). Ziel der Promotionsstrategie ist es, eine positive Wahrnehmung des Netzwerks in 
der Öffentlichkeit zu erzeugen als auch neue Mitglieder für das Netzwerk zu gewinnen. Je nach Zielsetzung des Netzwerks kann es verschiedene strategische Stoßrichtungen hinsichtlich der Mitgliederakquise geben. Im Sinne einer übergreifenden Expansionsstrategie sind Maßnahmen zur aktiven Akquise neuer Mitglieder zu verfolgen. Liegt der Fokus eher auf einer Stabilisierung der bestehenden Mitgliederbasis, kann eine passive Strategie verfolgt werden und damit verbunden eine sehr selektive Ansprache potenzieller neuer Mitglieder. Daneben ist auch die Reduktion der Anzahl der Mitglieder als Strategie denkbar, wenn dies der übergreifenden Strategie entspricht: Beispielsweise um den Verwaltungsaufwand zu vermindern und fokussierter mit aktiven Mitgliedern die Netzwerkziele zu verfolgen. Um die potenziellen Mitglieder geeignet ansprechen zu können, ist eine grundsätzliche Aufgabe im Rahmen der Netzwerkpromotion die Definition und Kommunikation des Nutzenversprechens an potenzielle Mitglieder. Sowohl den Interessenten als auch den bestehenden Mitgliedern gilt es, die Nutzenvorteile aus der Netzwerkmitgliedschaft individuell verständlich zu kommunizieren, um Anreize für den Eintritt und das Fortführen der Mitgliedschaft aufzuzeigen (Fitterer und Rohner 2010; Boughzala und Bououd 2013; Tapia 2009).

Für die Öffentlichkeitsarbeit des Netzwerks, ist es zudem wichtig, den Bekanntheitsgrad durch verschiedene Marketingmaßnahmen zu steigern. Hierfür eignen sich zum Beispiel Beiträge in regionalen Zeitungen und Fachzeitschriften, die Teilnahme an relevanten Messen und Kongressen sowie die Organisation eigener Veranstaltungen. Die Netzwerkpromotion zielt weiterhin darauf $a b$, sich mit den wichtigen regionalen und externen Interessengruppen zu vernetzen, um die Rahmenbedingungen für das Netzwerk positiv zu beeinflussen. So hat es sich in der Praxis bewährt, gute Kontakte mit öffentlichen Einrichtungen und zu politischen Akteuren auf regionaler Ebene und Landesebene zu pflegen (Speth und Zimmer 2015). Oftmals ergeben sich daraus finanzielle Förderungen, Projektkooperationen oder Begünstigungen, beispielsweise durch die Bereitstellung von Räumlichkeiten oder anderen Ressourcen für die Netzwerkarbeit. Durch den Einfluss von politischen Interessen kann es jedoch vorkommen, dass die eigenen Netzwerkziele weniger stark verfolgt werden können.

Zusammenfassend gilt es festzuhalten, dass durch Berücksichtigung der Kernfunktionen des Netzwerkmanagements die Zusammenarbeit im Netzwerk verbessert und nachhaltig auf eine langfristige Existenz ausgerichtet werden kann. Dabei bestehen zwischen den vier Funktionen der Netzwerkinfrastruktur, Netzwerkgovernance, Netzwerkmoderation und Netzwerkpromotion Interdependenzen, die sich bedingen und gegenseitig beeinflussen. Deshalb sollte keine der Funktionen isoliert betrachtet werden, sondern immer als Teil eines ganzheitlichen Netzwerkmanagementansatzes. Darüber hinaus ist es wichtig zu berücksichtigen, dass die Netzwerkmoderation das operative, zentrale Management widerspiegelt und viele der Kernaufgaben in der operativen Umsetzung ausgestaltet. 
Für das Netzwerkmanagement gilt es zu bedenken, dass durch den digitalen Wandel in der Gesellschaft viele der genannten Aufgabenfelder durch digitale Systeme durchgeführt oder unterstützt werden können. Gerade bei Netzwerkstrukturen, bei denen die einzelnen Mitglieder weniger häufig die Möglichkeit zum persönlichen Austausch haben, können digitale Systeme unterstützen, indem sie Arbeitsabläufe vereinfachen und zur engeren, schnelleren und besseren Netzwerkarbeit führen.

\subsection{Digitalisierung in der Netzwerkarbeit}

Besonders bei Prozessen mit vielen beteiligten Leistungserbringern, wie es bei Gesundheitsnetzwerken der Fall ist, stellt die Digitalisierung im Sinne eines zielgerichteten Einsatzes von Informations- und Kommunikationstechnologien eine mögliche Form der Optimierung dar (Lux 2017). Dieser Wandel wird durch den Begriff der digitalen Transformation beschrieben. Die Entwicklung kann als eine „... durchgängige Vernetzung aller Wirtschaftsbereiche und [die] Anpassung der Akteure an die neuen Gegebenheiten der digitalen Ökonomie“ bezeichnet werden (Bouée und Schaible 2015). Bisher werden die Potenziale dieser Technologien jedoch noch nicht hinreichend im Gesundheitsbereich eingesetzt (Lux 2017). So besteht beispielsweise bei Arztpraxen oder Krankenhäusern noch entscheidender Aufholbedarf (BITKOM und Fraunhofer ISI 2012). Deshalb ist es gerade im Gesundheitswesen aufgrund der fünf Millionen Beschäftigten, die im ständigen Austausch von Patientendaten miteinander stehen, wichtig, Netzwerke mit dem technologischen Einsatz von intelligenten Informations- und Kommunikationssystemen zu verbinden (BMG 2018). Digitale Technologien können unterstützende Treiber sein, diese Vernetzung umzusetzen, und stellen daher einen wichtigen Erfolgsfaktor für die Etablierung effizienter Prozesse und Strukturen in Netzwerken dar. Um das Netzwerkmanagement mit seinen Kernfunktionen zu verbessern, ist es von Vorteil, digitale Technologien, wie beispielsweise Kollaborationssysteme, Plattformen oder Datenbankensysteme, einzusetzen. Im Folgenden wird für die vier Kernfunktionen des Netzwerkmanagements aufgezeigt, welche Aufgaben durch den Einsatz von digitale Technologien unterstützt werden können. Bei der Auswahl entsprechender Software ist generell auf die Konformität hinsichtlich relevanter Datenschutz- und Datensicherheitsverordnungen zu achten als auch auf die individuelle Netzwerkstruktur und deren Heterogenitätsgrad in der Mitgliederstruktur.

Anhand von praktischen Beispielen werden in den folgenden Abschnitten Möglichkeiten aufgezeigt, wie einfache digitale Tools das Netzwerkmanagement unterstützen und entlasten können. Abb. 23.2 gibt einen Überblick darüber, wie die Kernaufgaben des Netzwerkmanagements sinnvoll digital unterstützt werden können. Insgesamt lässt sich feststellen, dass zahlreiche Informationssysteme und digitale Angebote für gemeinnützige Organisationen, wie bspw. eingetragene Vereine, kostenfrei oder kostenreduziert beziehbar sind. 

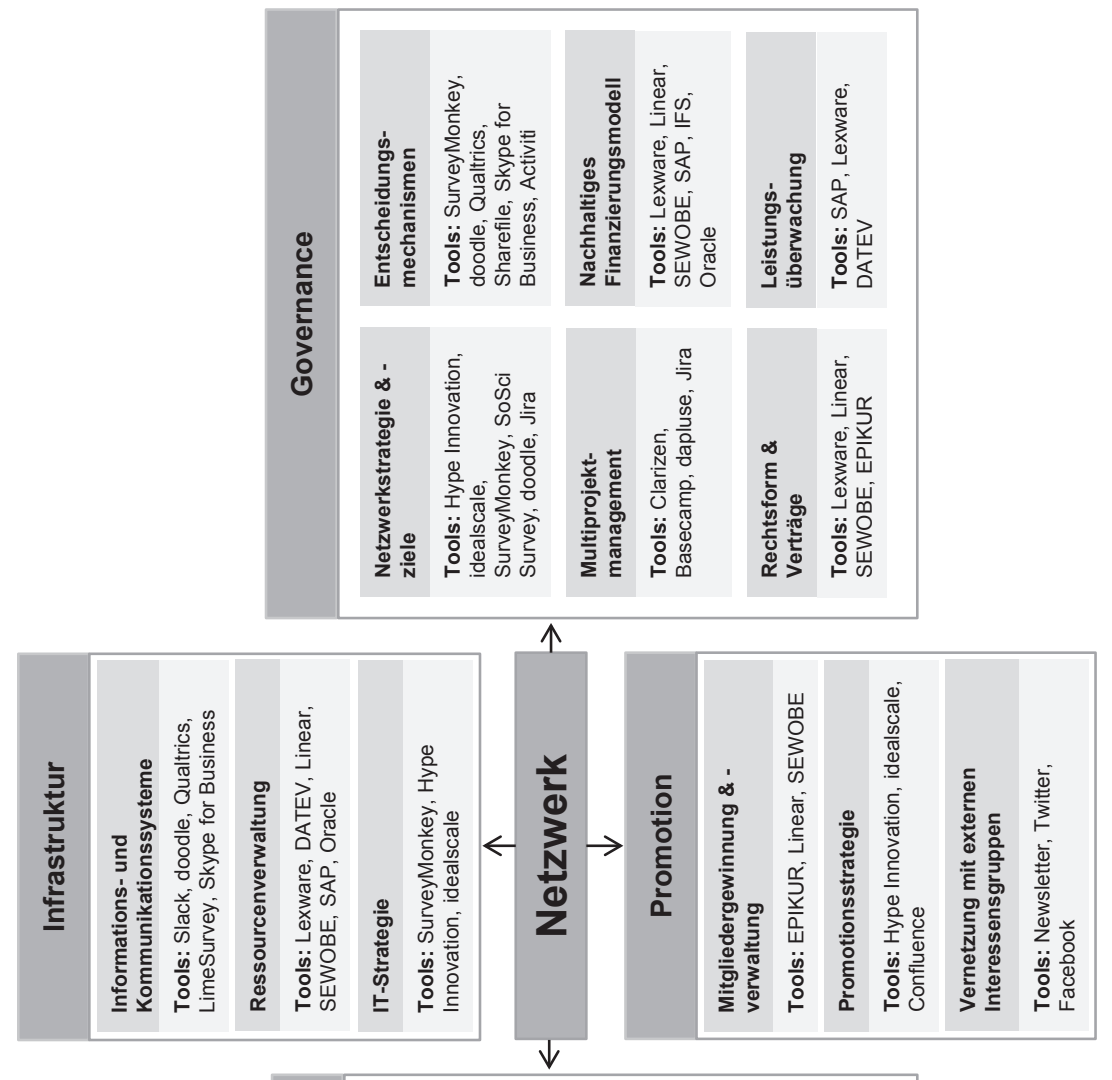

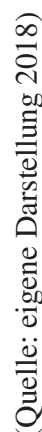

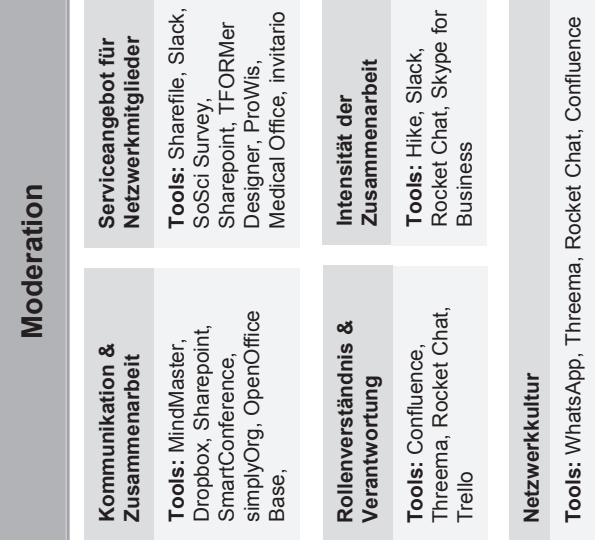




\subsubsection{Netzwerkinfrastruktur - Möglichkeiten zur digitalen Unterstützung der Basis für das Netzwerkmanagement}

Die Aufgaben der Netzwerkinfrastruktur sind, die finanziellen, personellen und physischen Ressourcen zu verwalten, eine IT-Strategie $\mathrm{zu}$ entwickeln und geeignete Informationssysteme und Kommunikationskanäle für das Netzwerk auszuwählen (Fitterer und Rohner 2010; Boughzala und Bououd 2013; Tapia 2009; Blondiau et al. 2013). Da die Netzwerkinfrastruktur die Basis für eine funktionierende Netzwerkarbeit bildet, sollte diese an den übergreifenden Zielen des Netzwerks ausgerichtet sein. Dabei ist es sinnvoll, einzelne Aufgaben durch unterschiedliche digitale Technologien zu unterstützen. Beispielsweise ist es möglich, für den Aufgabenbereich der Finanzverwaltung und Ressourcensteuerung unterschiedliche Typen von Informationssystemen einzusetzen. So kann einfache Software für die Finanzbuchhaltung (z. B. Lexware, DATEV, eBilanz-Online) neben der klassischen Buchhaltung den Zahlungsverkehr abwickeln, einen unkomplizierten und sicheren Datenaustausch mit bspw. dem Steuerberater unterstützen und bei der Erstellung finanzieller Berichte (z. B. Jahresabschluss) helfen (Hansen und Neumann 2009; Laudon et al. 2016). Nachdem Netzwerke in der Praxis oftmals als eingetragene Vereine organisiert sind, bietet sich auch der Einsatz einer umfassenderen, cloud-basierten Vereinsverwaltungssoftware (z. B. Linear, SEWOBE) an (Golinsky 2018). Diese Art von Software unterstützt bei allen zentralen Aufgaben von Vereinen, wie beispielsweise Beitragsmanagement, Buchführung, Mitgliederkommunikation, Zahlungsverkehr und auch Mitgliederverwaltung. So bieten derartige Systeme unter anderem Funktionalitäten zum Onlinebanking inkl. Lastschriftverfahren zum Einzug von Mitgliedsbeiträgen oder zur automatisierten Erstellung von Spendenbescheinigungen. Eine noch umfangreichere Softwareunterstützung stellen Enterprise-Resource-Planning-(ERP-)Systeme (z. B. SAP, IFS, Oracle) dar, die sich insbesondere zum Einsatz bei größeren Netzwerken eignen können. Mittels ERP-Systeme können die wirtschaftlichen und technischen Ressourcen geplant, gesteuert, überwacht und dokumentiert werden (Kessel und Vogt 2018). ERP-Systeme bestehen aus mehreren Komponenten, unterstützen die operativen Prozesse aller betrieblichen Funktionsbereiche (z. B. Finanzen, Personal, Material, Vertrieb) und arbeiten mit einer zentralen Datenbank (z. B. Data Warehouse). Bei ERP-Systemen ist jedoch zu berücksichtigen, dass sie durch ihren großen Funktionsumfang vergleichsweise teuer in der Anschaffung und Nutzung sind und sich die Einführung relativ aufwendig gestaltet und mehrere Jahre dauern kann.

Eine weitere Aufgabe im Rahmen der Netzwerkinfrastruktur ist es, geeignete Informationssysteme zur Kommunikation und zum Abwickeln der Netzwerkarbeit bereitzustellen und die Entwicklung einer passenden IT-Strategie für das Netzwerk zu etablieren. Zur gemeinschaftlichen Entwicklung der Strategie können Tools für Onlinebefragungen (z. B. SurveyMonkey, SoSci Survey, doodle, Qualtrics, LimeSurvey) genutzt werden, um über Mitgliederbefragungen Input zu strategischen Überlegungen und Wünschen der Netzwerkmitglieder einzusammeln. Die Befragungstools können zahlreiche bilaterale Gespräche ersetzen und folglich organisatorischen Aufwand 
reduzieren. Dadurch können sie einen wertvollen Beitrag für die Netzwerkinfrastruktur leisten. Es handelt sich dabei primär um Webapplikationen, die über den Browser aufgerufen werden können. Zur Erstellung einfacher Umfragen sind in der Regel keine Programmierkenntnisse erforderlich.

Eine weitere Möglichkeit für die digitale Datenerhebung ist der Einsatz von spezieller Software für Ideen- und Innovationsmanagement (z. B. Hype Innovation, ideascale). Diese Art von Software kann das Netzwerkmanagement ebenfalls bei der Entwicklung von Strategien unterstützen. Neben der einfachen Abfrage nach Input kann mithilfe des Tools auch kollaborativ an der Generierung von Ideen und Innovationen mit verschiedenen Inputgebern (intern und extern) gearbeitet werden. Die Ideen können mit der Zielgruppe direkt abgestimmt und durch sie bewertet werden. Darüber hinaus unterstützt Software für Ideen- und Innovationsmanagement auch bei der Umsetzung der neu entwickelten Ideen. Folglich kann die Software das Netzwerkmanagement bei der Verwaltung eines komplexen Ideen- und Innovationsportfolios unterstützen, indem sie die systematische Generierung, Verfolgung und Umsetzung neuer Ideen vereinfacht. Sie fördert außerdem die Innovationskultur im Netzwerk, da sie alle Akteure des Netzwerks als Inputgeber berücksichtigt und in den Innovationsprozess integriert.

\subsubsection{Netzwerkgovernance - Vereinfachte Steuerung des Netzwerks durch technologische Unterstützung}

Um die Kernfunktionen der Netzwerkgovernance, wie die Strategieentwicklung, Zielfestlegung, Entscheidungsmechanismen, Projektmanagement und Leistungsüberwachung, zu unterstützen, können digitale Technologien eingesetzt werden, um eine höhere Vernetzung und effizientere Arbeitsweisen zu erreichen.

Die Strategieentwicklung sollte gemeinschaftlich und unter Einbezug aller Mitglieder erfolgen, weshalb - analog zur Entwicklung der IT-Strategie - Tools für Onlinebefragungen (z. B. SurveyMonkey, SoSci Survey, doodle, Qualtrics, LimeSurvey) genutzt werden können, um über Mitgliederbefragungen Input zu strategischen Überlegungen einzusammeln. Auch Vereinsverwaltungssoftware (z. B. Linear, SEWOBE) bietet Funktionalitäten zur Durchführung von Mitgliederbefragungen (Golinsky 2018). Tools für Meinungsabfragen erleichtern ebenso Entscheidungsfindungsprozesse, indem beispielsweise bei größeren Investitionsentscheidungen vorab zu Entscheidungssitzungen Meinungen aller relevanten Akteure eingeholt und für die Sitzungen konsolidiert werden können, um die Diskussion effizienter und zielführender zu gestalten. Dadurch können zahlreiche persönliche Treffen und bilaterale Gespräche ersetzt und so der Aufwand für die Netzwerkgovernance reduziert werden. Für die Entwicklung einer übergreifenden Netzwerkstrategie ist zudem Software für das Ideen- und Innovationsmanagement (z. B. Hype Innovation, ideascale) relevant. Sie kann bei der kollaborativen Generierung, Bearbeitung und Umsetzung von Ideen helfen und den Prozess der Strategieentwicklung begleiten. Ein beispielhaftes Anwendungsszenario ist die innovative Gestaltung eines 
Telemedizinservices, bei dem neben verschiedenen Mitgliedsärzten auch Patienten in die Ideenfindung eingebunden werden.

Neben der Unterstützung bei kreativen Aufgaben können IT-Lösungen auch bei standardisierten und repetitiven Arbeitsvorgängen (sog. Workflows) helfen. So können Workflowmanagementsysteme (z. B. Activiti, ArtistaFlow) bei wiederkehrenden Entscheidungsprozessen (z. B. regelmäßige Budgetentscheidungen) die Netzwerkgovernance durch eine automatisierte Steuerung von Geschäftsprozessen unterstützen (Hansen und Neumann 2009; Laudon et al. 2016). Es werden Dokumente, Informationen und Aufgaben nach vorher bestimmten Regeln weitergeleitet, notwendige Daten und Anwendungen für den jeweiligen Bearbeitungsschritt zur Verfügung gestellt und Fristen sowie Ausnahmesituationen überwacht (Hansen und Neumann 2009). Dabei kann der Workflow bezüglich der Bearbeitungsreihenfolge und -bedingungen fest vorgegeben oder flexibel sein (Hansen und Neumann 2009).

Die Steuerung und Durchführung multipler Netzwerkprojekte ist ein weiterer Aufgabenbereich der Netzwerkgovernance. Hierfür kann Projektmanagementsoftware (z. B. Clarizen, Basecamp, dapulse, Jira) eingesetzt werden (Burghardt 2013). Diese Art der Softwareunterstützung erleichtert die Durchführung einzelner Projekte sowie die Verwaltung von Projektportfolios und liefert dadurch einen großen Mehrwert und eine starke Arbeitserleichterung für die Netzwerkgovernance. Grundsätzlich bieten Projektmanagementtools Funktionen für die Planung und Kollaboration zu den Projekten (Burghardt 2013). Die Planung des Projektleiters wird häufig durch To-do-Listen, Funktionen zur Arbeitseinteilung und -zuweisung sowie zur Meilensteinplanung unterstützt. Daneben wird die Software auch durch alle involvierten Projektmitarbeiter genutzt, beispielsweise um Arbeitsaufgaben einzusehen und Arbeitsstände zu dokumentieren. Zur Kollaboration werden Kommunikationsmöglichkeiten via Foren, Chats oder Whiteboards angeboten und eine gemeinsame Dokumentenablage bereitgestellt. Dies bietet den Vorteil, sich direkt und unkompliziert zu projektbezogenen Themen auszutauschen und dadurch längeren E-Mail-Verkehr zu vermeiden. Die verfügbaren Softwarelösungen bieten oftmals eine zusätzliche Integration weiterer Anwendungen, wie z. B. MicrosoftOffice-Programme oder Filesharingsysteme (Sharefile, Slack, Wire) an.

Je nach Größe und wirtschaftlicher Ausrichtung des Netzwerks können zur Überwachung und Dokumentation der Leistungserbringung einzelner Mitglieder und des Netzwerks als Ganzes ERP-Systeme (z. B. SAP, IFS, Oracle) einen Beitrag leisten, indem sie Informationen $\mathrm{zu}$ den wirtschaftlichen und technischen Ressourcen vorhalten (Kessel und Vogt 2018). Indem ERP-Systeme die erforderliche Datenbasis für entsprechende Kennzahlen liefern, unterstützen sie eine systematische und standardisierte Ausführung dieser Aufgaben. Dadurch kann die Evaluationsfunktion der Netzwerkgovernance und die Kontrolle der Leistungserbringung sinnvoll unterstützt werden. Im Gesundheitswesen sind als Kennzahlen insbesondere Zahlen zur Messung der Entwicklung der Gesundheit in einer bestimmten Region oder Population denkbar, zum Beispiel die Anzahl bestimmter Krankheiten oder die Anzahl bestimmter kurativer Maßnahmen. Im Netzwerkkontext sind aber auch Kennzahlen zur Leistungsüberprüfung des 
Netzwerkmanagements relevant. Für diesen Zweck kommen beispielsweise Erfüllungsgrade einzelner Projekte oder Netzwerkziele (z. B. Mitgliederfluktuation) und monetäre Erfolgszahlen, beispielsweise Gewinne, die bei Veranstaltungen erzielt wurden, infrage. Neben ERP-Systemen kann, gerade bei kleineren Netzwerken, spezielle Controllingsoftware (z. B. DATEV, eGECKO) zur kennzahlenbasierten Leistungsüberwachung eingesetzt werden, um die Netzwerkgovernance zu unterstützen (Hansen und Neumann 2009; Kessel und Vogt 2018). Durch den Einsatz von Controllingsoftware kann die wirtschaftliche Entwicklung des Netzwerks kontinuierlich überwacht werden, indem zum Beispiel Umsätze und Kosten beobachtet und Abweichungen von Plan- und Istzahlen grafisch dargestellt werden. Controllingsoftware hilft weiterhin bei der Erstellung von Berichten (z. B. Quartalsbericht).

Bei vielen Ärztenetzen ist der Einsatz von Praxisverwaltungssoftware (z. B. EPIKUR, medatixx) zur digitalen Verwaltung von Patienten- bzw. Behandlungsdaten als verpflichtend vorausgesetzt, insbesondere um Verträge zur integrierten Versorgung (IV-Verträge) mit den Krankenkassen zu ermöglichen (Busse et al. 2013; Schurr et al. 2009). Nur durch die digitale Verwaltung der Behandlungsdaten ist eine Leistungsüberwachung im Sinne dieser Verträge abbildbar und die Übermittlung der Daten an die Krankenkassen möglich. Insbesondere in Ärztenetzen ist der Einsatz eines Data Warehouse zur Aggregation und Integration von großen operativen Datenmengen sinnvoll, um eine systematische Leistungsüberwachung und -analyse zu ermöglichen. So kann eine bessere Grundlage für Entscheidungen und wertvoller Input für die Entwicklung von Strategien geschaffen werden.

\subsubsection{Netzwerkmoderation - Koordinierung und operative Organisation vereinfachen}

Um die strategischen Ziele zu verfolgen und operativ umzusetzen, bedarf es einer guten Netzwerkmoderation. Gerade der Austausch und die Kommunikation zwischen der strategischen Ebene, der Netzwerkgovernance, und der ausführenden Netzwerkmoderation können durch den sinnvollen Einsatz digitaler Tools verbessert werden. Zu den Aufgabenbereichen der Netzwerkmoderation gehören das Anbieten von Shared Services zur Entlastung der Netzwerkmitglieder, die Koordination der Kommunikation und Zusammenarbeit sowie Maßnahmen zur Förderung der Intensität der Zusammenarbeit und die Schaffung einer wertstiftenden Netzwerkkultur (Cuenca et al. 2013; Boughzala und Bououd 2013; Tapia 2009; Blondiau et al. 2013; Srai et al. 2013).

Die Bearbeitung sämtlicher interner und externer Anfragen sowie die netzwerkinterne Koordination von Projekten, Terminen und Netzwerktreffen stellen zentrale Aufgaben der Netzwerkmoderation dar. Die Nutzung von E-Mail-Software (z. B. Microsoft Outlook, Mozilla Thunderbird) ist bei der Bewältigung dieser kommunikationsintensiven Aufgaben der Standard für die Kommunikation. E-Mail-Software ermöglicht den Versand von Einzelnachrichten an einzelne Empfänger, den Versand von Sammelnachrichten 
an definierte Verteilerkreise und bietet in der Regel Funktionalitäten zum Verwalten von Kalendern und Terminen. Es besteht weiterhin die Möglichkeit, beim Versand von E-Mails Anhänge (z. B. Bild-, Text- oder Audiodateien) mitzuschicken und eine Priorität zur enthaltenen Nachricht anzugeben. Dabei liegen jedoch Einschränkungen bei der Größe der Dateianhänge und bei der Sicherheit und dem Datenschutz vor. Während die maximale Größe von Dateianhängen pro E-Mail auf ca. $20 \mathrm{MB}$ begrenzt ist (abhängig von den E-Mail-Providern), läuft die Übermittlung von E-Mails vom Absender zum Empfänger in den meisten Fällen über unverschlüsselte Internetverbindungen ab. Es besteht somit die Gefahr, dass die E-Mail-Inhalte von unbefugten Dritten mitgelesen werden, was insbesondere bei sensiblen, personenbezogenen Daten zu gesundheitlichen Themen besonders kritisch ist. Um die Sicherheitsprobleme zu eliminieren, kann eigens für die sichere Kommunikation innerhalb eines beschränkten Netzwerks entwickelte E-Mail-Software verwendet werden. Um die Einschränkungen bei der maximalen Größe für Dateianhänge zu beheben, bietet sich Filesharingsoftware an (e.g. Sharefile, Slack, Wire), um größere Dateien abzulegen und für alle Netzwerkmitglieder zugänglich zu machen. Jedoch handelt es ich bei den genannten Systemen um asynchrone Kommunikationskanäle. Für die Netzwerkarbeit ist es allerdings auch wichtig, im engen Austausch mit den Mitgliedern zu stehen. Dafür sollten Informationssysteme eingesetzt werden, die die Kommunikation und die Intensität der Zusammenarbeit fördern. Instant-Messenger-Dienste, wie zum Beispiel Slack, Hike, Threema oder Rocket Chat, welche die klassischen Kurznachrichtenformate SMS und MMS weitgehend ersetzt haben und Echtzeitkommunikation zwischen einzelnen sowie mehreren Personen ermöglichen, können hier wertvolle Unterstützung leisten (Hansen und Neumann 2009; Laudon et al. 2016). Sie ermöglichen schnelle Abstimmungen, erleichtern die Koordination der Zusammenarbeit und tragen so zu einer zügigeren Entscheidungsfindung bei. Darüber hinaus fördert die Echtzeitkommunikation den unkomplizierten, aktiven und direkten Austausch zwischen einzelnen Netzwerkmitgliedern und führt so zu einer höheren Intensität der Zusammenarbeit und letztlich zu einem stärkeren Zusammenhalt im Netzwerk. Der große Vorteil liegt in der Ermöglichung eines sehr schnellen und unkomplizierten Austauschs, der primär über mobile Endgeräte genutzt wird. Beispielsweise können sich Haus- und Fachärzte auf diesem Weg direkt zu einer Behandlung beraten. Neben dem Austausch von Textnachrichten und Dokumenten (z. B. Bild- oder Videodateien) können bei den meisten Diensten zusätzlich Telefonie- und Videotelefoniefunktionalitäten genutzt werden. Somit bündelt Instant Messaging eine Vielzahl an Austauschmöglichkeiten in Echtzeit. Unter der Voraussetzung einer stabilen Internetverbindung kommt es bei Textnachrichten zu keiner zeitlichen Verzögerung. Beim Versand von beispielsweise Audio- oder Videodateien kann es - in Abhängigkeit der Internetverbindung - zu kürzeren Ladezeiten kommen. Bei einzelnen Diensten können Einschränkungen bei der Einhaltung von Datensicherheit und -schutz vorliegen. Es gibt jedoch spezielle Dienste (z. B. Threema, Hike) die einen besonderen Fokus auf Sicherheits- und Datenschutzaspekte legen, sich auf den geschäftlichen Einsatz spezialisiert haben und daher auch für den Austausch sensibler Informationen geeignet sind. 
Die Koordination der Kommunikation und Zusammenarbeit im Netzwerk kann weiterhin durch digitale Telekonferenzsysteme (z. B SmartConference, easyAudio) erleichtert werden, indem sie eine ortsunabhängige Durchführung von Besprechungen ermöglichen. Aus interner Netzwerkperspektive fördern sie den regelmäßigen Austausch der Netzwerkakteure (z. B. von Arbeitsgruppen) und steigern so die Intensität der Zusammenarbeit und den Aufbau von Vertrauensbeziehungen. Daneben können Telekonferenzsysteme aber auch für die Kommunikation mit Externen genutzt werden, beispielsweise zur Durchführung von Bewerbungsgesprächen potenzieller Netzwerkmitglieder. Telekonferenzsysteme, die primär in Telefon- und Videokonferenzen unterteilt werden können, ermöglichen eine gemeinsame Erarbeitung und Diskussion von Themen über größere Entfernungen hinweg (Hansen und Neumann 2009; Laudon et al. 2016). Die meisten Telekonferenzsysteme bieten die zusätzliche Funktion einer simultanen Bearbeitung gemeinsamer Dokumente (z. B. Präsentation oder virtuelles Whiteboard), was dann als Dokumentenkonferenz bezeichnet wird (Hansen und Neumann 2009; Laudon et al. 2016). Sie ermöglichen eine standortübergreifende Audio- oder audiovisuelle Kommunikation zwischen einzelnen oder mehreren Personen und können mehrere Standorte in einer Besprechung zusammenführen. Die Nutzung von Videokonferenzsystemen (z. B. Skype for Business, LifeSize) kann in der Geschäftswelt heutzutage als Standard betrachtet werden. Ihr Einsatz in einem Besprechungsraum erfordert unter anderem das Vorhandensein eines Mikrofons, eines Lautsprechers und einer Kamera. Mit steigenden Anforderungen an die Übertragungsqualität sind höhere Ausgaben für die technische Ausstattung einzuplanen. Die meisten Videokonferenzsysteme bieten eine verschlüsselte Übertragung der Kommunikation, wobei es Einschränkungen gibt, beispielsweise beim Einwählen aus dem Festnetz (Hansen und Neumann 2009).

Die Kommunikation und Zusammenarbeit im Netzwerk erfordern interne Plattformen zum Austausch und zur gemeinsamen Bearbeitung und zur zentralen Ablage von Dokumenten. Neben Filesharingsoftware können auch Content-Management-Systeme (z. B. WordPress, Sharepoint) zur Verwaltung von Intranetseiten und gemeinsamen Dokumenten eingesetzt werden. Sie werden auch Redaktionssysteme genannt und dienen der kollaborativen Verwaltung von Beiträgen in einem Repositorium. Dabei besteht die Möglichkeit, Inhalte nur für gewisse Gruppen innerhalb des Netzwerks zugänglich zu machen (z. B. Beirat, Aufsichtsrat), sowie die Integration mehrerer Informationsquellen und die Weitergabe von Inhalten an Dritte (Hansen und Neumann 2009; Laudon et al. 2016).

Ein weiterer wichtiger Aufgabenbereich der Netzwerkmoderation ist die Bereitstellung von Dienstleistungen für die Netzwerkmitglieder, um diese im Sinne eines Shared Service Center in ihrer Arbeit zu entlasten. Um das Angebot an Dienstleistungen an den Bedürfnissen der Mitglieder auszurichten, können Befragungstools zur Durchführung von Bedarfsanalysen eingesetzt werden. Die Netzwerkmoderation ist interessiert am Input der Mitglieder und kann daher, beispielsweise um Feedback einzuholen, Tools zur Mitgliederbefragung einsetzen (z. B. SurveyMonkey, SoSci Survey, Qualtrics). Dadurch können die angebotenen Shared Services verbessert und Anregungen 
einholt werden. Ist das Netzwerk durch die Netzwerkgovernance als Verein organisiert, kann Vereinsverwaltungssoftware (z. B. Linear, SEWOBE) durch ihre Funktionalitäten zur Durchführung von Mitgliederbefragungen und zur Kommunikation mittels Kurznachrichten (SMS), Newsletter und Serienbriefe die Kommunikation und Kontaktpflege im Netzwerk vereinfachen und so die Netzwerkmoderation unterstützen (Golinsky 2018). Bei der Planung und Durchführung von Veranstaltungen können Onlinetools (z. B. invitario, simplyOrg) die Netzwerkmoderation bei der operativen Organisation von Veranstaltungen unterstützen, indem sie die Verwaltung der Anmeldungen erleichtern. Insbesondere bei größeren Veranstaltungen (z. B. Messen, Kongresse) helfen sie beispielsweise bei der Planung zum Umfang des Caterings und zur inhaltlichen Gestaltung des Programms. Diese Tools werden als Onlineformulare im Internetbrowser zum Beispiel auf der eigenen Homepage implementiert.

Ein großer Teil der Aufgaben der Netzwerkmoderation besteht aus Dokumentations- und Berichtswesen. Gerade bei der Dokumentation von Ergebnissen aus Arbeitsgruppen und Besprechungen (z. B. Protokolle) können digitale Tools eingesetzt werden. Hierzu gibt es spezielle Softwarelösungen (z. B. OpenOffice Base, TFORMer Designer, Confluence) zur Bewältigung von Dokumentations- und Berichtspflichten. Bei der gemeinschaftlichen Ablage von Gesprächsprotokollen oder internen Kontaktlisten kann die Netzwerkmoderation weiterhin durch Filesharingsoftware (z. B. Sharefile, Slack, Wire) unterstützt werden. Diese Dienste werden zur Onlinedatenspeicherung sowie zum Onlinedatenaustausch zwischen mehreren Personen genutzt. Der Zugriff auf diese Dienste läuft in der Regel über einen Internetbrowser oder über Apps ab. Der große Vorteil dieser Dienste ist eine schnelle und unkomplizierte Handhabung. Bei sensiblen Daten, wie es im Gesundheitsbereich häufig der Fall ist, ist darauf zu achten, dass die Cloud-Dienste den rechtlichen Vorgaben hinsichtlich Datensicherheit und Datenschutz entsprechen (z. B. bzgl. des Serverstandortes).

Bei der Verwaltung des Netzwerkwissens, das in den unterschiedlichen Arbeitsgruppen, Netzwerktreffen, Projekten, Besprechungen und Entscheidungsgremien entsteht, kann die Netzwerkmoderation durch Wissensmanagementsoftware (z. B. ProWis, Salesforce) unterstützt werden. Sie dient zur digitalen Verwaltung des Netzwerkwissens und umfasst alle organisatorischen und technischen Maßnahmen zur Erzeugung, Weitergabe, Speicherung und Auffindung des Wissens (Hansen und Neumann 2009; Laudon et al. 2016). Einige Systeme, wie z. B. oben genannte Content-Management-Systeme oder andere Dokumentenmanagementsysteme, übernehmen oftmals Teilaspekte des Wissensmanagements (Hansen und Neumann 2009; Laudon et al. 2016). Auch Tools, um Ideen und Konzepte zu strukturieren (z. B. MindMeister), bieten sich gerade für Arbeitsgruppen an, um Inhalte mittels einer Mindmap zu strukturieren und zu visualisieren. Dabei ist der Vorteil für die Netzwerkmoderation, einen schnellen Überblick zu bekommen und zu erfassen, wer aktuell an welchen Themen arbeitet und wie weit sie vorangeschritten sind.

Zur Förderung der Kommunikation und Zusammenarbeit im Netzwerk können bei Ärztenetzen spezielle digitale Technologien eingesetzt werden. So spielt Software 
zur Realisierung einer praxisübergreifenden Vernetzung (z. B. Medical Office, Connected Care, elVi) eine wichtige Rolle, um einen digitalen Datenaustausch (inkl. Videosprechstunde) zu ermöglichen (Schurr et al. 2009). Diese Art der Software zielt darauf ab, Arztpraxen und weitere Akteure aus dem Gesundheitssektor informations- und kommunikationstechnisch zu vernetzen, um eine integrierte Versorgung zu ermöglichen. So wird die tägliche Arbeit der Ärzte effizienter sowie effektiver und Behandlungsfehler können häufiger vermieden werden. Dabei können die einzelnen Ärzte ihre eigenen Praxisverwaltungssysteme beibehalten, weil diese in die vernetzte Lösung integriert werden. Ärztenetze und andere Gesundheitsnetze streben darüber hinaus häufig eine elektronische Netzpatientenakte an, um eine effizientere, integrierte Behandlung von Patienten zu realisieren (Heinze und Bergh 2017; Landrock und Gadatsch 2018). Zur Ermöglichung von elektronischen Netzpatientenakten wird spezielle Software (z. B. Medical Office Simple Practice) eingesetzt (Heinze und Bergh 2017).

\subsubsection{Netzwerkpromotion - Außendarstellung durch digitale Medien verbessern}

Neben der Organisation der internen Strukturen und Prozesse des Netzwerkmanagements gilt es auch, das Netzwerk nach außen darzustellen. Damit die Netzwerkarbeit eine Reichweite und positive Aufmerksamkeit in der Öffentlichkeit bekommt, ist es wichtig, eine erfolgreiche Netzwerkpromotion zu etablieren. Die Aufgabenbereiche der Netzwerkpromotion umfassen die Entwicklung einer Promotionsstrategie, die Gewinnung und die Auswahl neuer Mitglieder sowie die Vernetzung mit externen Interessengruppen.

Zur gemeinschaftlichen Entwicklung einer Promotionsstrategie können ebenfalls Tools für Onlinebefragungen (z. B. SurveyMonkey, SoSci Survey, doodle, Qualtrics, LimeSurvey) genutzt werden, um Input zu strategischen Überlegungen einzuholen. Auch Vereinsverwaltungssoftware (z. B. Linear, SEWOBE) bietet Funktionalitäten zur Durchführung von Mitgliederbefragungen (Golinsky 2018). Für die Entwicklung einer Promotionsstrategie ist außerdem - analog zum Einsatz für die IT-Strategie - Software für das Ideen- und Innovationsmanagement (z. B. Hype Innovation, ideascale) relevant. All diese Tools entlasten das Netzwerkmanagement bei der Bewältigung seiner Aufgaben für die Promotion des Netzwerks.

Für die digitale Kommunikation mit externen Interessengruppen kann die Netzwerkpromotion über E-Mail-Software unterstützt werden, indem beispielsweise potenzielle Mitglieder via Mailing auf das Netzwerk aufmerksam gemacht werden oder Pressemitteilungen und Newsletter zu den Netzwerkaktivitäten an einen externen Interessentenkreis verteilt werden. Instant-Messenger-Dienste (z. B. WhatsApp, Threema, Rocket Chat) können als Kontaktpunkt für externe Anfragen genutzt werden, um so die Netzwerkpromotion bei der schnellen Bearbeitung von Anfragen externer Interessengruppen zu unterstützen. Außerdem können mithilfe von Instant-Messenger-Diensten (z. B. durch 
ein Abonnement des WhatsApp-Dienstes durch die Netzwerkmitglieder) Informationen zu den Netzwerkaktivitäten im Text-, Audio- oder Videoformat regelmäßig an einen breiten Verteilerkreis gestreut werden.

Auch Content-Management-Systeme (z. B. Sharepoint, WordPress) unterstützen die Netzwerkpromotion bei der Verwaltung der Netzwerkwebseiten (Hansen und Neumann 2009; Laudon et al. 2016). Für die Promotion der Netzwerkaktivitäten und zur Vermarktung des Netzwerks nach außen kann außerdem Social-Networking-Software (z. B. Twitter, Facebook) eingesetzt werden. Diese sind mittlerweile in der Bevölkerung weitverbreitet und können vom Netzwerkmanagement zur Unterstützung der Netzwerkpromotion eingesetzt werden, um relevante Informationen zu den Netzwerkaktivitäten (z. B. zu konkreten Veranstaltungen oder neuen Publikationen) an eine breite Öffentlichkeit zu verteilen und so Aufmerksamkeit für die eigenen Aktivitäten zu generieren. Über Facebook können beispielsweise auch Veranstaltungen angelegt, beworben und organisiert werden. In der Regel legt sich die Organisation in diesen Systemen ein eigenes Profil an, hat eine eigene Profilseite und kann dort verschiedene Dateiformate (z. B. Text, Audio, Video) veröffentlichen (Corduan 2018; Linke 2015). Interessenten können anschließend darauf reagieren, indem sie die geteilte Information weiterleiten, kommentieren oder ihre Zustimmung oder Abneigung durch entsprechende Buttons ausdrücken (Corduan 2018; Linke 2015; Hippner und Wilde 2005). Der große Vorteil von Social-Networking-Software ist die breite Öffentlichkeitswirkung und die Möglichkeit zum direkten Austausch. Auf der anderen Seite birgt ihre Nutzung die Gefahr negativer Eigendynamiken, die schnell unkontrollierbar werden können. Wird beispielsweise ein unvorteilhafter Beitrag von vielen Personen aufgegriffen und negativ kommentiert, kann sich in der Öffentlichkeit sehr schnell ein negatives Bild zur Organisation ergeben (Richter 2014).

Für die Außendarstellung werden oftmals Netzwerkberichte, Jahresberichte und weitere Publikationen verfasst. Hier können spezielle Softwarelösungen zur Bewältigung von Dokumentations- und Berichtspflichten (z. B. OpenOffice Base, TFORMer Designer) zu Hilfe genommen werden und die Netzwerkpromotion dadurch entlasten.

Ein weiterer Aufgabenbereich der Netzwerkpromotion, der durch Informationssysteme unterstützt werden kann, ist die Mitgliederverwaltung. Diese Aufgaben können von Vereinsverwaltungssoftware (z. B. Linear, SEWOBE) unterstützt werden (Golinsky 2018), die unter anderem Funktionalitäten zur Mitgliederverwaltung beinhaltet und beispielsweise eine digitale Kontaktdatenpflege ermöglicht.

\subsection{Schlussbetrachtung}

Ein Lösungsansatz, um die Problematiken, die aktuell im Gesundheitswesen bestehen, zu adressieren, ist es, Netzwerke zu etablieren, die unterschiedliche Akteure zusammenbringen, um dadurch eine bessere Versorgung unterstützen zu können. Die sinnvolle Ausgestaltung und strategische Entwicklung der vier Kernbereiche Netzwerkinfrastruktur, 
Netzwerkgovernance, Netzwerkmoderation und Netzwerkpromotion können das Netzwerkmanagement verbessern und helfen, Netzwerke nachhaltig zu verstetigen. Dabei ist es wichtig, die einzelnen Bereiche auch integriert zu betrachten und die Zusammenhänge der einzelnen Ebenen zu verstehen.

Die Digitalisierung kann eine zusätzliche sinnvolle Unterstützung für die Herausforderungen, die im Gesundheitswesen bestehen, sein, besonders wenn es sich um vernetzte Strukturen handelt. Bisher werden diese aktuell jedoch noch zu wenig genutzt. Betrachtet man die vielen Möglichkeiten der digitalen Tools, die die Netzwerkarbeit unterstützen und auch verbessern können, zeigt sich das große Potenzial im Gesundheitsbereich, das durch die Digitalisierung besteht. Gerade wenn das Zusammenspiel der Arbeitsabläufe mit dem IT-System und der Prozessentscheidungsfindung logisch und verständlich erscheint, ist der Einsatz digitaler Technologien erfolgreich (Penter und Pfaffner 2017). Die angeführten Beispiele zur digitalen Vernetzung stellen keine abschließende Liste dar und sollen lediglich Inspiration dazu geben, geeignete digitale Tools für die Netzwerkarbeit auszuwählen. Netzwerkmanager sollten ihre Arbeitsweisen des klassischen Netzwerkmanagements reflektieren und überlegen, welche Art von Tool für die Ziele und Aufgaben des Netzwerks eine Unterstützung darstellen kann. Grundsätzlich gilt, dass es nicht das Ziel sein sollte, möglichst viele der genannten Beispiele zur digitalen Unterstützung einzusetzen, sondern sich aufgrund der Aufgaben und der Komplexität des Netzwerks zu überlegen, welche der digitalen Technologien sinnvoll sind und wie diese zu implementieren sind. Wichtig ist dabei, genau zu prüfen, welche der genannten Tools für das Netzwerk passend sind, da dies immer von der Größe und auch der Homogenität bzw. Heterogenität der Mitglieder anhängig ist. Vor diesem Hintergrund sind auch Investitionsentscheidungen für Informationssysteme zu berücksichtigen. Hierbei sind einmalige Kosten, laufende Kosten und Pfadabhängigkeiten zu hinterfragen.

Des Weiteren ist auch anhand der Strategie abzuwägen, welcher Grad an Digitalisierung im Netzwerkmanagement sinnvoll ist. Trotz des Einsatzes unterschiedlicher digitaler Technologien, gerade bei der Kommunikation unter den Mitgliedern und im Austausch mit dem Netzwerkmanagement, ist es dennoch unerlässlich, persönliche Treffen durchzuführen. Der direkte und persönliche Kontakt lässt sich durch Informationstechnologie nicht ersetzen. Digitale Technologien können allerdings zu intensiverem Austausch führen und somit die Zusammenarbeit als auch die Netzwerkkultur stärken.

Obwohl die Digitalisierung viele Vorteile für die Netzwerkarbeit im Gesundheitswesen mit sich bringen kann, gibt es auch Herausforderungen, welche berücksichtigt werden müssen. Bei der Implementierung neuer digitaler Software und Systeme gilt es, die Nutzer einzubeziehen und zu schulen (Penter und Pfaffner 2017). Individuen tendieren dazu, nicht immer offen gegenüber Innovationen und Veränderungen zu sein. Deshalb ist es wichtig, dass die eingeführten Tools von allen Mitgliedern akzeptiert und angenommen werden und sich beispielsweise als Standardkommunikations- und -informationskanäle etablieren. Demnach kann es auch kontraproduktiv sein, wenn zu viele unterschiedliche Systeme implementiert werden. Des Weiteren gilt es, dem Thema Datenschutz und Datensicherheit besondere Aufmerksamkeit zu schenken. Gerade bei 
den sensiblen und persönlichen Gesundheitsdaten ist hierbei ein Maximum an Datenschutz nötig, um das Vertrauen und die Akzeptanz der Patienten zu erhalten (Leikert 2017). Unterstützt wird der Schutz der persönlichen Daten durch die seit 2018 geltende Datenschutz-Grundverordnung (DSGVO). Auf die strengeren Vorgaben und höheren Strafen, die die DSGVO mit sich bringt, sollte gerade bei Netzwerken, wenn unterschiedliche Leistungserbringer miteinander agieren, ein besonderes Augenmerk gelegt werden. Daher ist es empfehlenswert, einen externen Datenschutzbeauftragten für das Netzwerk zu engagieren. Des Weiteren ist eine strategische Verankerung von IT-Compliance-Konzepten notwendig. Es ist für das Netzwerk nicht ausreichend, sich allein auf die IT-Sicherheitsvorkehrungen der Softwareanbieter zu verlassen. Daher zusätzlich dezentral und anwendungsbezogen Maßnahmen ergriffen werden, um den Datenschutz und die Datensicherheit gewährleisten zu können (Mleczeck 2017).

\section{Literatur}

Aschhoff M (2017) Herausforderung Interoperabilität - Standards für die digitale Gesundheitsversorgung. In: Matusiewicz D, Pittelkau C, Elmer A (Hrsg) Die Digitale Transformation im Gesundheitswesen. Transformation, innovation, disruption. Medizinisch Wissenschaftliche Verlagsgesellschaft GmbH \& Co. KG., Berlin, S 41-43

Aumiller J (2014) Unter-, Fehl- und Überversorgung. MMW - Fortschritte der Medizin 156(14):75

Becke G, Bleses P, Frerichs F, Goldmann M, Hinding B, Schweer MKW (2016) Zusammen - Arbeit Gestalten. Soziale Innovationen in sozialen und gesundheitsbezogenen Dienstleistungen. Springer Fachmedien, Wiesbaden

Beer M, Wolf T, Garizy TZ (2015) Systemic risk in IT portfolios - an integrated quantification approach. Int Conf Inf Syst 36:1-18

Beermann M (2017) Politische Perspektiven für die Zukunft der digitalen Gesundheit. In: Matusiewicz D, Pittelkau C, Elmer A (Hrsg) Die Digitale Transformation im Gesundheitswesen. Transformation, innovation, disruption. Medizinisch Wissenschaftliche Verlagsgesellschaft GmbH \& Co. KG, Berlin, S 36-40

BITKOM, Fraunhofer ISI (2012) Gesamtwirtschaftliche Potenziale Intelligenter Netze in Deutschland. https://www.bitkom.org/Bitkom/Publikationen/Gesamtwirtschaftliche-Potenziale-intelligenter-Netze-in-Deutschland.html. Zugegriffen: 28. Juli 2018.

Blondiau A, Cleven A, Rohner P (2013) Towards a hospital cooperation maturity model. Construction and evaluation of a maturity model and a corresponding tool for the identification of challenges and success factors for cooperation in the hospital sector. Int J Health Inf Manage Res 1(1):438-448

BMG (2018) E-Health - Digitalisierung im Gesundheitswesen. https://www.bundesgesundheitsministerium.de/e-health-initiative.html\#c2846. Zugegriffen: 28. Juli 2018.

BMWi (2019) Gesundheitswirtschaft. Fakten \& Zahlen, Ausgabe 2018. Ergebnisse der Gesundheitswirtschaftlichen Gesamtrechnung. https://www.bmwi.de/Redaktion/DE/Publikationen/Wirtschaft/ gesundheitswirtschaft-fakten-zahlen-2018.pdf?_blob=publicationFile\&v=3. Zugegriffen: 12. Juni 2019.

Bönisch S (2017) Was bringt Vernetzung im Gesundheitswesen. Springer Fachmedien, Wiesbaden

Bouée C-E, Schaible S (2015) Perspektiven der digitalen Zukunft. https://bdi.eu/media/user_ upload/Digitale_Transformation.pdf. Zugegriffen: 28. Juli 2018. 
Boughzala I, Bououd I (2013) The development and application of a community maturity model. Int J Inf Technol Manage 12(3/4):273-297

Braeuninger D, Rakau O (2010) Gesundheitswirtschaft im Aufwind. https://www.wko.at/site/Plattform-Gesundheitswirtschaft/Studien—Publikationen/Studien/Gesundheitswirtschaft_Aufwind. pdf. Zugegriffen: 28. Juli 2018.

Burghardt M (Hrsg) (2013) Einführung in Projektmanagement. Definition, Planung, Kontrolle, Abschluss Siemens Aktiengesellschaft, 6. Aufl. Publicis Publishing, Erlangen

Busse R, Schreyögg J, Stargardt T (2013) Management im Gesundheitswesen. Das Lehrbuch für Studium und Praxis, 3. Aufl. Springer, Berlin

Corduan A (2018) Social Media als Instrument der Kundenkommunikation. Vergleichende Studie von Unternehmen in China, Deutschland und den USA. Springer Fachmedien. Wiesbaden. http://dx.doi.org/10.1007/978-3-658-22317-5. Zugegriffen: 28. Juli 2018.

Cuenca L, Boza A, Alemany MME, Trienekens JJM (2013) Structural elements of coordination mechanisms in collaborative planning processes and their assessment through maturity models. Application to a ceramic tile company. Comput Ind 64(8):898-911

Ernstberger A, Schmucker U, Herbst T, Nerlich M (2017) Verbesserung der Prozessqualität bei Traumapatienten durch digitale Bild und Dokumentenübermittlung. In: Pfannstiel MA, Da-Cruz P, Mehlich H (Hrsg) Digitale Transformation von Dienstleistungen im Gesundheitswesen II, Impulse für das Management. Springer Gabler, Wiesbaden, S 89-106

Fiedler E (2018) Überversorgung im Gesundheitswesen. https://www.versicherungsmagazin.de/ lexikon/ueberversorgung-im-gesundheitswesen-1946885.html. Zugegriffen: 28. Juli 2018.

Fitterer R, Rohner P (2010) Towards assessing the networkability of health care providers. A maturity model approach. Inf Syst E-Bus Manage 8(3):309-333

Gelauff GMM, den Broeder C (1997) Governance of stakeholder relationships. The German and Dutch experience, Bd 1. Société universitaire européenne de recherches financières, Amsterdam, S 1

Gerlinger T, Burkhardt W (2018) Bismarcks Erbe: Besonderheiten und prägende Merkmale des deutschen Gesundheitswesens. http://www.bpb.de/politik/innenpolitik/gesundheitspolitik/72553/deutsche-besonderheiten?p=all. Zugegriffen: 28. Juli 2018.

Golinsky F (2018) Moderne Vereinsorganisation. Vereinsmanagement leicht gemacht. Springer. Berlin. http://dx.doi.org/10.1007/978-3-662-56459-2. Zugegriffen: 28. Juli 2018.

Hansen HR, Neumann G (2009) Wirtschaftsinformatik, Bd 2669, 10. Aufl. Lucius \& Lucius, Stuttgart

Heinze O, Bergh B (2017) Persönliche einrichtungsübergreifende Gesundheits- und Patientenakten (PEPA) als zentrale Infrastrukturkomponente einer patientenzentrierten Gesundheitsversorgung. In: Müller-Mielitz S, Lux T (Hrsg) E-Health-Ökonomie. Springer Gabler, Wiesbaden, S $847-858$

Hippner H, Wilde T (2005) Social software. Wirtschaftsinformatik 47(6):441-444

Katz ML, Shapiro C (1985) Network externalities, competition, and compatibility. Am Econ Rev 75(3):424-440

Kessel T, Vogt M (2018) Wirtschaftsinformatik Schritt für Schritt. Arbeitsbuch, Bd 4430, 2. Aufl. UVK Verlagsgesellschaft, Konstanz

Landrock H, Gadatsch A (2018) Big Data im Gesundheitswesen. Begriffsabgrenzung und Einleitung. In: Landrock H, Gadatsch A (Hrsg) Big Data im Gesundheitswesen kompakt: Konzepte, Lösungen, Visionen. Springer Fachmedien, Wiesbaden, S 1-16

Laudon KC, Laudon JP, Schoder D (2016) Wirtschaftsinformatik. Eine Einführung, 3. Aufl. Pearson, Hallbergmoos

Leikert K (2017) eHealth - Wie die digitale Vernetzung unser Gesundheitssystem zukunftssicher macht. In: Matusiewicz D, Pittelkau C, Elmer A (Hrsg) Die Digitale Transformation im 
Gesundheitswesen. Transformation, Innovation, Disruption. Medizinisch Wissenschaftliche Verlagsgesellschaft GmbH \& Co. KG, Berlin, S 25-29

Linke A (2015) Management der Online-Kommunikation von Unternehmen. Steuerungsprozesse, Multi-Loop-Prozesse und Governance. Springer VS, Wiesbaden

Lux T (2017) Vernetztes Schmerzmanagement durch E-Health in der Pflege. In: Pfannstiel MA, Da-Cruz P, Mehlich H (Hrsg) Digitale Transformation von Dienstleistungen im Gesundheitswesen II: Impulse für das Management. Gabler, Wiesbaden, S 165-183

Mleczeck I (2017) Datenschutz -Beweggrund, Begleiter oder Bürde der digitalen Transformation. In: Matusiewicz D, Pttelkau C, Elmer A (Hrsg) Die Digitale Transformation im Gesundheitswesen, Transformation, Innovation, Disruption. Medizinisch Wissenschaftliche Verlagsgesellschaft GmbH \& Co. KG, Berlin, S 336-340

Penter V, Pfaffner K (2017) Digitale Gesundheit - Fluch oder Segen? In: Matusiewicz D, Pittelkau C, Elmer A (Hrsg) Die Digitale Transformation im Gesundheitswesen. Transformation, innovation, disruption. Medizinisch Wissenschaftliche Verlagsgesellschaft GmbH \& Co. KG, Berlin, S 303-306

Präventionsnetzwerk Ortenaukreis (2018) Was ist PNO? http://www.pno-ortenau.de/media/custom/2565_18_1.PDF?1480680072. Zugegriffen: 28. Juli 2018.

Richter A (Hrsg) (2014) Vernetzte Organisation. De Gruyter Oldenbourg, München. http://dx.doi. org/10.1524/9783486747317. Zugegriffen: 28. Juli 2018.

Robert Koch-Institut (2018) Chronische Erkrankungen. https://www.rki.de/DE/Content/Gesundheitsmonitoring/Themen/Chronische_Erkrankungen/Chronische_Erkrankungen_node.html. Zugegriffen: 28. Juli 2018.

Schubert H (2008) Netzwerkmanagement. Koordination von professionellen Vernetzungen: Grundlagen und Praxisbeispiele. VS Verlag, Wiesbaden

Schurr M, Dumont M, Kunhardt H (2009) Unternehmen Arztpraxis - Ihr Erfolgsmanagement. Aufbau, Existenzsicherung, Altersvorsorge. Springer, Berlin

Schwarze J (2014) Projektmanagement mit Netzplantechnik, 11. Aufl. NWB Verlag, Herne

Simon M (2011) Das Gesundheitssystem in Deutschland. Eine Einführung in Struktur und Funktionsweise, 3. Aufl. Huber, Bern

Speth R, Zimmer A (Hrsg) (2015) Lobby work. Interessenvertretung als Politikgestaltung, Bd 47. Springer VS, Wiesbaden

Srai JS, Alinaghian LS, Kirkwood DA (2013) Understanding sustainable supply network capabilities of multinationals. A capability maturity model approach. In: Proceedings of the Institution of Mechanical Engineers, Part B: Journal of Engineering Manufacture 227(4):595-615.

Stabell CB, Fjeldstad ØD (1998) Configuring value for competitive advantage. On chains, shops, and networks. Strategic Manage J 19(5):413-437

Stadtelmann M, Lindner A, Woratschek H (2015) Wertschöpfungskonfigurationen. In: Woratschek H, Schröder J, Eymann T, Buck M (Hrsg) Wertschöpfungsorientiertes Benchmarking. Logistische Prozesse in Gesundheitswesen und Industrie. Springer, Berlin, S 25-32

Statista (2018) Bevölkerung in Deutschland nach Altersgruppen in den Jahren 2013, 2030 und 2060 (in Millionen). https://de.statista.com/statistik/daten/studie/71539/umfrage/bevoelkerung-in-deutschland-nach-altersgruppen/. Zugegriffen: 28. Juli 2018.

Stiefelhagen P (2018) Gibt es eine Überversorgung älterer Diabetespatienten? Info Diabetologie 12(3):44

Sydow J (2010) Management von Netzwerkorganisationen - zum Stand der Forschung. In: Sydow J (Hrsg) Management von Netzwerkorganisationen. Beiträge aus der „Managementforschung“, 5. Aufl. Gabler \& GWV Fachverlage GmbH, Wiesbaden, S 373-470

Sydow J, Duschek S (2013) Netzwerkzeuge. Tools für das Netzwerkmanagement. Springer Fachmedien, Wiesbaden 
Tapia RS (2009) ICoNOs MM: the IT-Enabled collaborative networked organizations maturity model. In: Camarinha-Matos LM, Afsarmanesh H, Paraskakis I (Hrsg) Leveraging knowledge for innovation in collaborative networks. 10th IFIP WG 5.5 Working conference on Virtual Enterprises, PRO-VE 2009, Thessaloniki, Greece, October 7-9, 2009. Proceedings, Bd 307. Springer, Berlin, S 307.

Teller M, Longmuss J (2007) Netzwerkmoderation. Netzwerke zum Erfolg führen. Ziel Verlag, Augsburg

Tiemeyer E (2017) Handbuch IT-Management. Konzepte, Methoden, Lösungen und Arbeitshilfen für die Praxis, 6. Aufl. Hanser, München

Wasmuth T (2013) Gesundheitsausgaben. Determinanten und Auswirkungen auf die Gesundheit; theoretische Modellierung und empirische Analyse, Bd. 66. Lang, Frankfurt a. M.

Weatherly JN (2013) Netzwerkmanagement im Gesundheitsbereich. In: Luthe E-W (Hrsg) Kommunale Gesundheitslandschaften. Springer VS, Wiesbaden, S 103-117

Dr. Christoph Buck ist wissenschaftlicher Assistent am Lehrstuhl Wirtschaftsinformatik der Universität Bayreuth und in der Fraunhofer Projektgruppe Wirtschaftsinformatik des Fraunhofer FIT. Nach dem Diplomstudium der Betriebswirtschaftslehre an der Universität Bayreuth legte er seine Promotion an der Rechts- und Wirtschaftswissenschaftlichen Fakultät der Universität Bayreuth ab. Seine Forschungsschwerpunkte sind die digitale Transformation, Information Privacy, E-Health und datengetriebene Geschäftsmodelle.

Simone Burster ist wissenschaftliche Mitarbeiterin am Lehrstuhl Wirtschaftsinformatik der Universität Bayreuth. Nach ihrem Masterstudium in Internationaler Wirtschaft und Governance begleitet sie seit 2017 das vom BMBF geförderte Projekt Innovative Netzwerke für Dienstleistungen und Gesundheit in Regionen von morgen (INDiGeR) am Lehrstuhl Wirtschaftsinformatik der Universität Bayreuth. Ihre Forschungsschwerpunkte sind Gesundheitsnetzwerke sowie Information Privacy.

Serkan Sarikaya ist wissenschaftlicher Mitarbeiter am Kernkompetenzzentrum Finanz- \& Informationsmanagement. Nach seinem Diplomstudium in informationsorientierter Betriebswirtschaftslehre an der Universität Augsburg startete er 2013 mit seiner Promotion am Kernkompetenzzentrum Finanz- \& Informationsmanagement. Im Rahmen seiner Forschungsaktivitäten befasst er sich schwerpunktmäßig mit interkulturellen Kundenbeziehungen.

Julia Thimmel ist studentische Mitarbeiterin am Lehrstuhl Wirtschaftsinformatik der Universität Bayreuth. Nach ihrem Bachelorstudium begann sie ihr Masterstudium in Gesundheitsökonomie an der Universität Bayreuth.

Prof. Dr. Torsten Eymann ist Inhaber des Lehrstuhls Wirtschaftsinformatik der Universität Bayreuth und stellvertretender wissenschaftlicher Leiter der Projektgruppe Wirtschaftsinformatik des Fraunhofer FIT. Nach dem Diplomstudium der Informatik an der Universität Mannheim legte er seine Promotion an der Albert-Ludwigs-Universität Freiburg ab. Seit dem Jahr 2004 hat Prof. Dr. Eymann den Lehrstuhl für Wirtschaftsinformatik an der Universität Bayreuth inne. Seit dem Jahr 2015 ist er Vizepräsident der Universität Bayreuth für Informationstechnologie und Entrepreneurship. Seine Forschungsschwerpunkte sind IT-Security, E-Health und die digitale Transformation. 